\title{
The Ontology based FMEA of Lead Free Soldering Process
}

\author{
Martin Molhanec, Pavel Mach, David Asamoah Bamfo Mensah \\ Department of Electro-Technology, Faculty of Electrical Engineering \\ Czech Technical University in Prague \\ Czech Republic \\ molhanec@fel.cvut.cz
}

\begin{abstract}
Paper is directed to improvement of a FMEA (Failure Mode and Effects Analysis) procedure in the field of reflow lead free soldering by the use of an ontology paradigm. Some ontology editor tools, suitable for our intention, are presented. Particular phases of ontology utilization for development of more exact approach to the FMEA procedure of reflow lead free soldering are analyzed. A proposal of a user's guide for the FMEA procedure based on an ontology paradigm is presented. This user's guide is included in university courses Management of Production Quality and Complex Quality Control provided by the Department of Electro-Technology.
\end{abstract}

\section{INTRODUCTION}

Since the early 1980s, the world of quality has been bombarded with the concept of "continual improvement". For most of us, this concept has been focused on "prevention" as opposed to "appraisal." The push for this continual improvement makes "FMEA" a dynamic document, changing as a system, design, process, product and/or service changes with the intent always to make a better system, design, process, product, and/or service.

By definition, the FMEA is a methodology to maximize the satisfaction of the customer by eliminating and/or reducing known or potential problems.

A Failure Mode And Effect Analysis (FMEA) is an engineering method used to define, identify and eliminate a known/or potential problems, errors and so on from the system, design, process or service before they reach the customer or It is a methodology to maximize the satisfaction of the customer by eliminating and /or reducing known or potential problems. To do this the FMEA must begin as early as possible.

It is the most widely used analysis procedure in practice at the initial stage of the system development and usually performed during the conceptual and initial design phase of the system in order to assure that all possible failure modes have been considered and that proper provision has been made to eliminate all the potential failures.

\section{FMEA COMPONENTS}

The essence of the FMEA is to identify and prevent known and potential problems from reaching the customer. To do that, assumptions have to be made, one of which is that problems have different priorities. There are three components that help define the priority of failures:

\footnotetext{
$\checkmark$ Occurrence (O)

$\checkmark$ Severity of effect (S)

$\checkmark$ Detection (D)
}

Occurrence is the assessment of the probability that the specific cause of the Failure mode will occur. It is part subjective, but the wording should describe the probability. Failure history is helpful in increasing the truth of the probability. Questions of the following type are helpful:

Severity is an assessment of the seriousness of the Effect and refers directly to the potential failure mode 
being studied. A customer in a FMEA process is both the internal and where appropriate, external Customer.

The severity ranking is also an estimate of how difficult it will be for the subsequent operations to be carried out to its specification in Performance, Cost, and Time.

Detection is an assessment of the probability that the proposed Process Controls will detect a potential cause of Failure or a Process weakness. Assume the Failure has occurred and then assess the ability of the Controls to prevent shipment of the part with that defect. Low Occurrence does not mean Low Detection - the Control should detect the Low Occurrence. Statistical sampling is an acceptable Control. Improving Product and/or Process design is the best strategy for reducing the Detection ranking Improving means of Detection still requires improved designs with its subsequent improvement of the basic design. Higher rankings should question the method of the Control.

Risk Priority Number is a mathematical product of the numerical Severity, Occurrence, and Detection ratings. This number is used to place priority on items than require additional quality planning.

$$
R P N=S . O . D
$$

\section{FMEA TYPES}

a. System FMEA - (called also a concept FMEA) is used to analyze of a system or a subsystem in the early concept at design stage. It focuses on potential failure modes between the functions of the system caused by the system deficiencies.

b. Design FMEA - is used to analyze of products before they are released to manufacturing. It focuses on failure modes caused by design deficiency.

c. Process FMEA - is used to analyze of manufacturing and assembly processes. A process FMEA focuses on failure modes caused by process or assembly deficiencies.

d. Service FMEA - is used to analyze of services before they reach the customer. A service FMEA focuses on failure modes (tasks, errors, mistakes) caused by system or process deficiencies.

\section{Start of a FMEA Program}

A FMEA program starts when the new system, designs, products, processes or services are designed.

When existing system, designs, products, processes or services are about to change regardless of reason.

$\checkmark$ When new applications are found for the existing condition of the system, designs, products, processes or services.

$\checkmark$ When improvements are considered for the existing system, designs, products, processes or services.

All FMEA processes follow these steps

$\checkmark$ Process Review.

$\checkmark$ Brainstorm Potential Failure Modes.

$\checkmark$ List Potential Effects of Each Failure Mode.

$\checkmark$ Assigning Severity, Occurrence and Detection Ratings.

$\checkmark$ Assign a Severity Rating for Each Effect.

$\checkmark$ Assign an Occurrence Rating for Each Failure Mode.

$\checkmark$ Assign a Detection Rating for Each Failure Mode and/or Effect.

$\checkmark$ Calculate the Risk Priority Number for Each Failure Mode.

$\checkmark$ Prioritize the Failure Modes for Action

$\checkmark$ Take Action to Eliminate or Reduce the High-Risk Failure Modes

\section{FMEA COMPLETING}

FMEA can be considered finished or completed when the system designs, products, processes or services are considered complete.

For FMEA types:

a. System FMEA may be considered finished when all the hardware has been defined and the design declared frozen.

b. Design FMEA may be considered finished when a release date for production has been set.

c. Process FMEA may be considered finished when all operations have been identified and evaluated and all critical and significant characteristics have been addressed in the control plan. 
d. Service FMEA may be finished when the design of the system and individual tasks have been defined and evaluated and all critical and significant characteristics have been addressed in the control plan [1].

\section{Problem Statement}

As we assert in [10] the lead free soldering is a joining technology, which substitutes, for the long time used, $\mathrm{Sn}-\mathrm{Pb}$ soldering. The reason is that $\mathrm{Pb}$ is not a nature friendly metal and therefore solders based on an alloy $\mathrm{Sn}-\mathrm{Pb}$ have been interdicted from the use since the year 2006. However, the use of new types of lead free solders is joined with new problems, e.g. with the use of new types of fluxes, with higher temperature of soldering, or with worst surface quality of the joints [9]. Therefore it is necessary to carry out a new FMEA analysis to find critical possible failures of lead free soldering process and their impact on quality of soldered joints.
The most frequent failures, which can occur in the process of lead free soldering, are as follows:

$\checkmark$ Too high soldering temperature can damage a mounted component or decrease its life time.

$\checkmark$ Improper surface finish (e.g. finish with low wettability) can cause worst mechanical and electrical properties of the joint.

$\checkmark$ Improper flux can cause worst mechanical and electrical properties of the joint.

$\checkmark$ Too high time of soldering can cause recrystalization of solder and decrease a soldered joint quality.

There are many other failures joined with this process instead above mentioned ones.

The process of FMEA is a rather bit complicated and therefore it seems to be effective to find some ontology, which will formalize it.

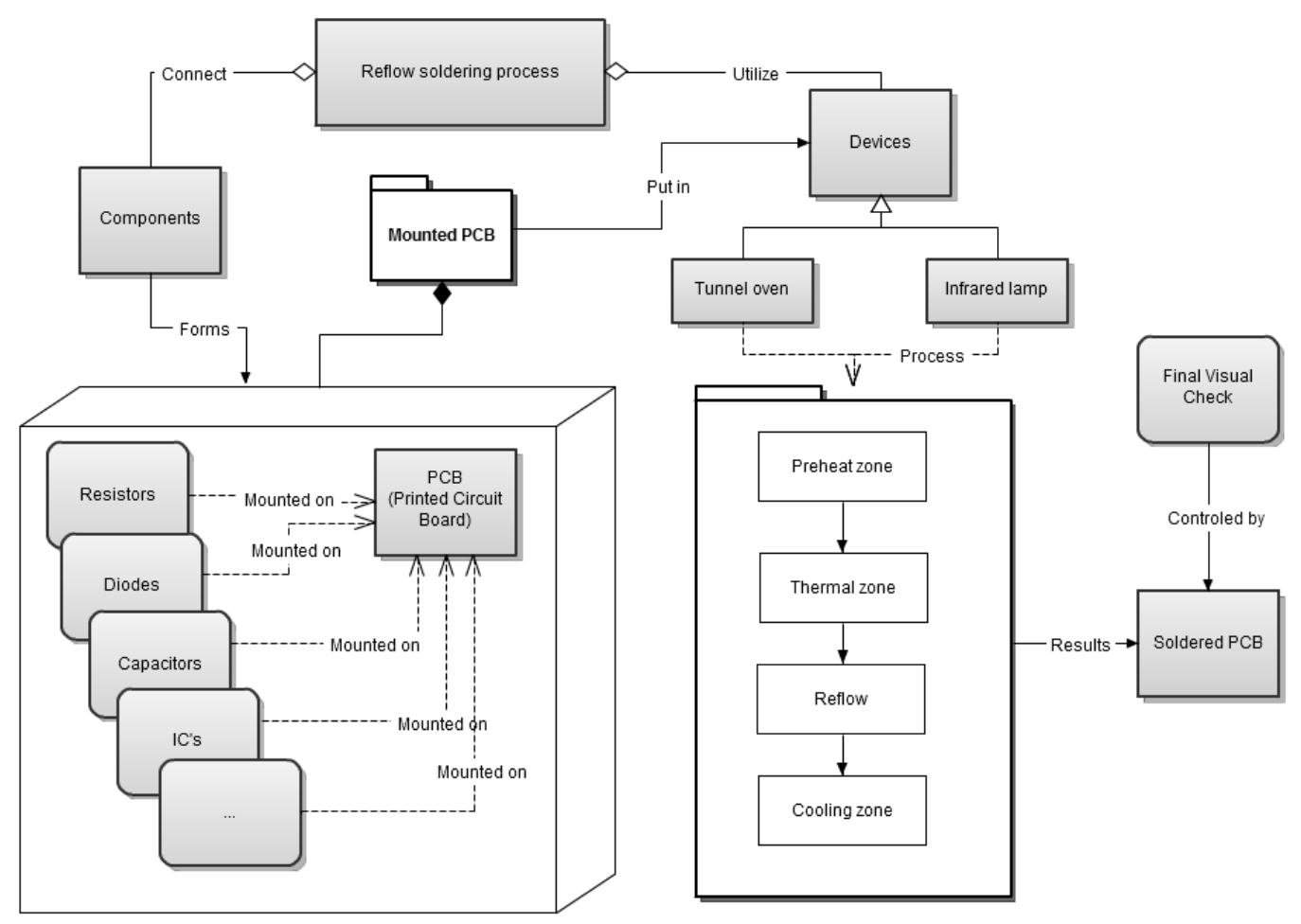

Fig. 1. Proposed ontology scheme for reflow soldering process. 


\section{OUR APPROACH}

The goal of the work is to verify if application of the ontological approach will help to solve problems mentioned in previous section. Our advancement will be composed from the following steps.

1. Selection of some PC-based FMEA SW tool, open and free preferably.

2. FMEA analysis of a lead-free soldering process without the use of ontology based approach.

3. Selection of a PC-based ontology editor suitable for application on FMEA, open and free preferably.

4. Literature study of process, product and defect ontology.

5. Design of ontology of soldering process under inspection.

6. The design of a suitable method for application of a FMEA analysis process based on ontology of a lead-free soldering process.

7. The application of above mentioned method on a lead free soldering process.

8. The comparison and evaluation of results found in steps 2 and 7.

9. Embodiment of this newly developed approach into courses oriented on quality education at our department.

Our approach is based on ontology paradigm. Ontology in philosophy is the study of the nature of being, existence or quality in general, as well as of the basic categories of being and their relations [2]. In our work we deal with the more specialization kind of ontology, i.e., the informatics or upper ontology [3].

We propose a strong support of the analysis process by performing ontology of FMEA domain. Basically ontology is a part of philosophy science, but for the case of our intention, we will use more simple definition.

As defined by Wikipedia: "Ontology is a formal representation of a set of concepts within a domain and the relationships between those concepts. It is used to reason about the properties of that domain, and may be used to define the domain".
As noted in [4] and [5], ontology can support the development and performance of an FMEA in two ways.

- First, it offers a common understanding of the concepts of the domain of our focus and the FMEA procedure ourselves as well.

- Second, the knowledge held in the ontology based model can be computationally processed.

Both these basic facts support the idea to use an ontological approach for improvement of the FMEA procedure.

According to [5] the ontological based system FMEA analysis is performed according to the following five main phases:

1) Structural analysis.

2) Functional analysis.

3) Migration of environmental agents.

4) Searching for degradations.

5) Selection and application of degradations.

This proposed method is very proper designed, but in our case of soldering process we need the method for the process, not for the system, analysis. On the other hand, the aforementioned method is a good inspiration for our achievement. The right design of the method performing the ontological based process FMEA analysis, the step 6 in previous text, is a key factor of our approach.

The main contribution of ontology based approach is a clear and consistent graphical description and visual presentation of all FMEA concepts and dependencies. More simply, the FMEA ontology performs a synergy effect for an analyst. Despite the fact that storage of all FMEA concepts and dependencies in form of the ontological model in computerised form allows us a computerized processing, searching and reporting of these facts. 


\section{Results}

A team of several young graduate students under the leadership of two experts, one in the field of soldering process and second in the field of enterprise ontology, was established in order to study the ontological approach in quality areas. This team also get a research grant targeted to this field.

The present state of our work is in the phase of performing steps 1 to 5 . A preliminary proposal of the soldering process ontology is shown in Fig. 1. But it is necessary to note that this is a starting period of the work in this problem.

According to our opinion mainstreaming of ontology approach in the FMEA procedure is a valuable contribution to the field of quality management in general. The results of this research have also been utilized in courses Management of Production Quality and Complex Quality Control provided by the department of Electro-Technology.

\section{ACKNOWLEDGEMENT}

This research (work) has been supported by Ministry of Education, Youth and Sports of Czech Republic under research programs "Quality of Electrical Energy", number MSM6840770017 and "Diagnostics of Materials", number MSM6840770021.

\section{REFERENCES}

[1] Asamoah Bamfo Mensah D.: "Failure and Effect Analysis of Electrically Conductive Adhesive Joining," Diploma thesis, CTU Prague, 2008

[2] Wikipedia, "Ontology":, Online: < http://en.wikipedia.org/wiki/Ontology>

[3] Wikipedia, "Ontology (information science)", Online: $<$ http://en.wikipedia.org/wiki/Ontology_(computer_sci ence) $>$

[4] Dittmann, L., Rademacher, T., Zelewski, S. 2004. Performing FMEA using ontologies. In: Kleer, J.; Forbus, D. (eds.): 18th International Workshop on Qualitative Reasoning, Evanston, Illinois. pp. 209-216.

[5] Ahmed Laaroussi, Bruno Fiès, Rémi Vankeisbelckt, Julien Hans, "Ontology-aided FMEA for construction products", in "Bringing ITC knowledge to work", 24th W78 Conference Maribor 2007, 26.-29.6.2007

[6] Protégé, editor of ontology, online: < http://protege.stanford.edu/>

[7] SWOOP, editor of ontology, online: < http://www.mindswap.org/2004/SWOOP>

[8] JOE, editor of ontology, online: $<$ http://www.cse.sc.edu/research/cit/demos/java/joe/>

[9] Mach, P., Duraj, A.: Failure Mode and Effects Analysis of a Process of Reflow Lead-Free Soldering. ISSE 2008, Budapest. 2008

[10] Mach, P. - Molhanec, M., The Ontology based FMEA of Lead Free Soldering Process, In: 32nd ISSE 2009 Proceedings [CD-ROM]. Brno: VUT v Brně, FEI, 2009, vol. 1, ISBN 978-1-4244-4260-7. 